\section{Importance of genomic studies for drug withdrawal with mental toxicities}

\author{
Da-Yong Lu, ${ }^{1}$ Ting-Ren Lu² \\ ${ }^{1}$ School of Life Sciences; ${ }^{2}$ College of \\ Science, Shanghai University, Shanghai
}

\begin{abstract}
Undesired side-effects and toxicities of drugs, especially in the area of new-drug development, are negligibleless, unpredicable and often disastrous once being encountered. The suicidal behavior caused by antidepressant treatment is a typical of clinical evidence recently being discovered. We previously hypothesized that patients' genetic status would decide the suicidal incident rate of antidepressants - it is pharmacogenetics of antidepressants may contribute of this toxicity in patients. In this review, we discuss this problem by comparing many strings of pharmacogenomics evidence of antidepressants recently being published with many other strings of evidence such as drug withdrawal with hepatotoxicity. We argue herein that pharmacogenetics may be very useful in drug withdrawal for mental toxicity. Because this is low-incidence toxicities, which are more reliable on human's genetic characteristics. We stress the importance of genomics studies for drug withdrawal in future.
\end{abstract}

\section{Introduction}

New drug development and licensing are large enterprising needing a lot of money or funds to support and a high risky job with an average successive rate approximately $7 \% .^{1-2}$ These characteristics make the high-price of new drugs in markets and hospitals. Drug withdrawal is another important factor to affect the interests of drug-manufacturing companies and further make drugs' production a high-risk job. All these processes in return result in high-payment for patients for prescribing these drugs. We can reasonably deduce that the price of drug development and licensing will decide the total cost of patients for prescribing these drugs. In this review, we will discuss the importance of genomic studies in drug withdrawals.

\section{Discussion}

Drugs withdraw often happen when some unexpected side-effects (toxicities) in clinics occur. Undesired side-effects and toxicities of drugs, especially in the area of new-drug development, are negligibleless, unpredicable and often disastrous once being encountered.3 There has been an old famous Chinese saying; each drug always inherits 30 percent toxicity comparing with its full range of effectiveness. Updating drug withdrawal system can be regarded as an effective means to save experimental or clinical resources and avoid the lost of effective drugs from the markets. ${ }^{3}$ There has been a hypothesis if pharmacogenomics could help rescue drug withdrawals from markets. ${ }^{4-5}$ Although some people considered that pharmacogenomic studies might not be an effective way to rescue drugs, ${ }^{4}$ we argued it might work in some circumstances, especially in neural toxicities. ${ }^{3,5}$ We initiate this argument from following evidence and findings: i) Unlike hepatotoxicities, the occurrences of neural toxicities are rare. That means only small amounts of patients show the neural toxicities by drugs, such as antibiotics-induced mania. ${ }^{6}$ This evidence can be easily supported by genetic or genomic explanations. ${ }^{3}$ ii) Some polymorphisms in patients have shown a highrisk of neural toxicities, such as antidepressant-induced suicides and (antibiotics) aminoglycoside-induced hearing impairment.

As for 2004, the US and European regulatory agencies began implementing verification programs to assess the influence on suicidal behavior from the use of antidepressants such as SSRIs, ${ }^{7-8}$ almost all antidepressants have been investigated and several antidepressants have been withdrawn from the markets. Soon after this investigation and panic, we propose a hypothesis that genetic might also play a role in the risk of antidepressant-induced suicide. ${ }^{5}$ So we indicate that we need not pay our attention only on drugs themselves, but we ought to diverse our attention into genetic study of clinic investigations. From our perspective, patients' genetic conditions might play more important roles than chemical structure of drugs in deciding the side-effects or toxicities of drugs. Though, it has not been widely received. However more and more clinical evidence is accumulating and somewhat supporting our hypothesis in drugs with mental toxicities. These kinds of clinical evidence can be concluded as follows through chronic orders.

It has been shown that one patient has a suicidal ideation or behaviors by using one type of antidepressants, he will more likely do so by using other types of antidepressants. ${ }^{9}$

The drug-induced suicidal ideation and behaviors will more easily occur in patients who have a genetic tendency of madness ideation and behaviors. ${ }^{10}$

Last but not least, more and more genetic markers and alleles have been found to link
Correspondence: Da-Yong Lu, School of Life Sciences, Shanghai University, Shanghai, 200444, PR China.

E-mail: ludayong@sh163.net

Key words: antidepressants, antibiotics, pharmacogenomics, drug withdrawal, mental toxicity, drug manufacture, suicidal ideation.

Received for publication: 27 May 2011.

Revision received: 3 August 2011.

Accepted for publication: 8 August 2011.

This work is licensed under a Creative Commons Attribution NonCommercial 3.0 License (CC BYNC 3.0).

(C) Copyright D-Y. Lu and T-R. Lu, 2011

Licensee PAGEPress, Italy

Drugs and Therapy Studies 2011; 1:e11

doi:10.4081/dts.2011.e11

with suicidal ideations and behaviors in clinics. ${ }^{11-19}$ The literature has shown this relation in drugs with mental toxicities. However, these are direct evidence which fully support the relationship between human's genetic features and drug toxicities. Extant pharmacogenomic studies of increases in suicidal ideation and behavior in antidepressant-treated depressed patients report associations with polymorphisms in genes involved in at least 9 related genes, including transcription (CREB1), neuroprotection (BDNF, NTRK2), glutamatergic and noradrenergic neurotransmission (GRIA3, GRIK2, ADRA2A), stress and inflammatory response (FKBP5, IL28RA) and synthesis of glycoproteins (PAPLN). ${ }^{17}$ These large volumes of polymorphism of genetic markers will greatly affect the quality and fairness of drug assessment and approval or withdraw. Thus we must pay special attention to it. There has also been reported that familial transmission of suicidal behavior. ${ }^{20}$ To conclude it is welcoming to broaden our horizon from just aiming at one or two types of antidepressants into understanding the whole situations from all these characters and relationship in between. To better argue with the idea that pharmacogenetics is basically useless in drug withdrawal, ${ }^{4}$ we here add that pharmacogenetics may be very useful in drug withdrawal for mental toxicity. This is low-incidence toxicities, which are more reliable on human's genetic characteristics. And since 2004, there is very few antidepressants have been withdrawn from markets. It seems pharmacogenetics only has little effects in drug withdrawal with hepatotoxicity. ${ }^{4}$

Future directions of these researches should aim at two important challenges. One is clinically individualized therapy in depressed patients. ${ }^{21}$ But at present, the detective prices 
prohibit its universal applications. ${ }^{22}$ The other challenge is how to perfect drug approval or withdraw system. ${ }^{3}$ This great program of environmental-genomic-aged-drug interaction network should be viewed as a wholesome singlemodular model of deciding drug destination. The single criteria of possibility can be roughly calculated as:

$$
\mathrm{P}_{\text {toxicity }}=\mathrm{P}_{\text {chemical }}+\mathrm{P}_{\text {genetic }}+\mathrm{P}_{\text {environment }}
$$

To conclude, the future drug evaluation, approving or disapproval systems should be more considerate with genetic or genomic basis of individuals rather than solely building on the average data of all populations. We should be forever readiness for any new initiative of drug approval systems and upgrading our understanding and knowledge about both features of drugs and shed new light from all these investigations.

\section{References}

1. Mervis J. Productivity counts - but the definition is key. Science (Washington DC) 2005;309:726-7.

2. Schein PS, Scheffler B. Barriers to efficient development of cancer therapeutics. Clin Cancer Res 2006;12:3243-8.

3. Lu DY, Lu TR, Zhu PP. Undesired neural side-effects of a drug, a chemical and genetic interrelated problem. Cent Nerv Syst Agents Med Chem 2010;10:108-12.

4. Shah RR. Can pharmacogenetics help rescue drugs withdrawn from the market? Pharmacogenomics 2006; 7:889-908.

5. Lu DY, Lu TR, Ding J. May genetic factors play a role in the risk of antidepressant- induced suicide. Med Hypotheses 2007;69: 1380-1.

6. Check E. Drug suicide risks prompt call for FDA action. Nature 2004;427:474.

7. Holden C. FDA weighs suicide risk in children on antidepressants. Science (Washington DC) 2004;303:745.

8. Rubino A, Roskell N, Tennis P, et al. Risk of suicide during treatment with venlafaxine, citalopram, fluoxetine, and dothiepin: retrospective cohort study. $\mathrm{Br}$ Med $\mathrm{J}$ 2007;334:242-7.

9. Kraus JE, Horrigan JP, Carpenter DJ, et al. Clinical features of patients with treatment-emergent suicidal behavior following initiation of paroxetine therapy. $\mathrm{J}$ Affect Disord 2010;120:40-7.

10. McMahon FJ, Buervenich S, Charney D, et al. Variation in the gene encoding the serotonine $2 \mathrm{~A}$ receptor is associated with outcome of antidepressant treatment. Am J Human Genet 2006;78:804-14.

11. Perlis RH, Purcell S, Fava M, et al. Association between treatment-emergent suicidal ideation with citalopram and polymorphisms near cyclic adenosine monophosphate response element bind protein in STAR*D study. Arch Gen Psychiatry 2007;64:689-97.

12. Lage G, Paddock S, Manji H, et al. Genetic markers of suicidal ideation emerging during citalopram treatment of major depression. Am J Psychiatry 2007;164: 1530-8.

13. Menke A, Lucae S, Kloiber S, et al. Genetic markers within glutamate receptors associated with antidepressant treatmentemergent suicidal ideation. Am J Psychiatry 2008;165:917-8.

14. Perround N, Aitchison KJ, Uher R, et al. Genetic predictors of increase in suicidal ideation during antidepressant treatment in the GENDEP project. Neuropsychopharmacology 2009;34:2517-28.

15. Laje G, Perlis RH, Rush AJ, McMahon FJ. Pharmacogenetics studies in STAR*D: strengths, limitations and results. Psychiatr Serv 2009;60:1446-57.

16. Brent DA, Melhem N, Ferrell R, et al. Association of FKBP5 polymorphisms with suicidal events in the Treatment of Resistant Depression in Adolescents (TORDIA) study. Am J Psychiatry 2010; 167:190-7.

17. Brent D, Melhem N, Turecki G. Pharmacogenomics of suicidal events. Pharmacogenomics 2010;11:793-807.

18. Kohli MA, Salyakina D, Pfennig A, et al. Association of genetic variants in the neurotrophic receptor-encoding gene NTRK2 and a lifetime history of suicide attempts in depressed patients. Arch Gen Psychiatry 2010;67:348-59.

19. Uher R, Perroud N, Ng MY, et al. Genomewide pharmacogenetics of antidepressant response in GENDEP project. Am J Psychiatry 2010;167:555-64.

20. Brent DA, Melhem N. Familial transmission of suicidal behavior. Psychiatr Clin North Am 2008;31:157-77.

21. Lin KM, Perlis RH, Wan YJ. Pharmacogenomic strategy for individualizing antidepressant therapy. Dialogues Clin Neurosci 2008;10:401-8.

22. Perlis RH, Patrick A, Smoller JW, Wang PS. When is pharmacogenetic testing for antidepressant response ready for the clinic? A cost-effectiveness analysis based on data from the $\mathrm{STAR}^{*} \mathrm{D}$ study. Neuropsychopharmacology 2009;34:2227-36. 